\title{
Effects of High-Sucrose and High-Saturated Fat Diets on Learning Abilities in Old Sprague-Dawley Rats
}

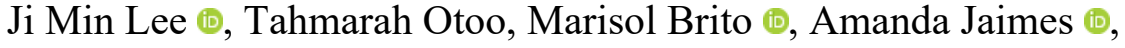 \\ Arlene Martinez, and Jennifer Trevitt (i)
}

California State University, Fullerton, Psychology Department

\begin{abstract}
Background and Purpose: With an increase in longevity, many studies have explored the influences of different lifestyle factors on successful aging. This study hypothesizes that older rats fed with a nutritionally balanced standard diet would perform better on learning tasks than rats fed with either a highsucrose or a high-saturated fat. It also hypothesizes that older rats fed with a high-sucrose diet would perform better than those fed with a high-saturated fat. Methods: The learning abilities of the 15 -month-old rats $(\mathrm{N}=36)$ were assessed by conducting forward and reverse learning tasks using a $\mathrm{T}$ maze apparatus. Results: The results showed that rats on a nutritionally balanced diet performed significantly better on both learning tasks than those on either the high-sucrose or the high-saturated fat (p's $<.05$ ). This may be due to the fact that the high-sucrose and high-saturated fat diets exacerbated a cognitive decline in geriatric rats. There was no significant difference between the learning abilities of the rats on a high-sucrose or high-saturated fat diet (p's $>.05)$. Conclusion: At an older age, both highsucrose and high-saturated fat diets have a similarly detrimental influence on cognitive health.
\end{abstract}

(C) 2021 and CC-BY 4.0 licensed by the authors.

Keywords: lifestyle habits, cognition, diet, learning abilities, successful aging, older adults

\section{Introduction}

With an overall increase in longevity, many healthcare professionals, researchers, gerontologists, and the general population have become interested in preserving older adults' physical and mental health. Such a process of ensuring physical and mental wellness in the older adult population is widely known as "successful aging." It is important to note that the concept of successful aging is not onedimensional but multi-dimensional and encompasses not only life expectancy, but also life satisfaction, social networks, physical health, mental health, cognitive function, spirituality, and more (Bowling \& Dieppe, 2005).
Previous research explored the impact of factors such as exercise, diet, and social interactions on longevity and overall health in older adults (Jacob et al., 2016). Among the factors affecting cognition in older adults, there is a great need to address the relationship between diet and cognition, particularly as dietary manipulations are a readily accessible way to impact the aging process positively. The topic of diet and its effects on cognition amongst older adults is important for many reasons, including changes in sugar and fat consumption over the past century ("Poor Nutrition," 2021). Research has found that the average annual consumption of refined sugar in the forms of high-fructose corn syrup went from $8.1 \mathrm{~kg} /$ person in the $19^{\text {th }}$ century to $65 \mathrm{~kg} /$ person in the twentieth century (Stephan et al., 2010). 
The researchers suggested that perhaps there is a link between the drastic increase in fructose intake and the noticeable increase in the number of Alzheimer's disease-diagnosed patients in the past century. In another study, researchers looked at the dietary pattern among older adults residing in rural areas (Bell et al., 2007). In comparison to those living in cities, older adults with chronic disease conditions in rural areas were more likely to consume high sugar and fat-modified foods such as artificially sweetened beverages, sweeteners, butter, margarine, and various hightrans-fat salad dressings. Bell et al. (2017) concluded that the discrepancies in the accessibility of healthy, organic foods and the lack of awareness of diet's effect on cognitive function could significantly alter the rate of cognitive decline in older adults. The results of these studies indicate an overall change in dietary patterns towards increased consumption of high sucrose and fat-modified foods amongst older adults and an increase in the rate of prevalence of neurodegenerative diseases. Although the link between diet and neurodegenerative diseases is inconclusive from these studies, they contribute to the importance and relevance of exploring the relationship between diet and cognition in older adults.

\section{Effects of Diet on Older Adult Cognitive Health}

Several studies have found that diets can have direct and indirect effects on physical and mental health. A recent longitudinal study found that a Mediterranean diet (MeDi), comprising low consumption of red meat, moderate consumption of fish and poultry, and high consumptions of nuts, fruits, and vegetables, improved cognition in older adults (Luciano et al., 2017). The MeDi emphasizes the intake of monounsaturated fats that are considered heart-friendly and found in nuts and plants, while it discourages the intake of saturated fats and sugar, which are considered unhealthy. Interestingly, the study found that it was not the individual components but the interactions of these components that improved cognitive function. In addition, other researchers found that the participants who had high adherence to MeDi showed greater conservation of the brain's structural connectivity than those who had low adherence to a MeDi (Samieri et al., 2015). The researchers stated that the difference in structural connectivity maintenance was associated with about a decade of delayed aging, indicating that the participants who followed the MeDi experienced slower rates of cognitive decline than those who did not. More broadly, all of the studies demonstrated that a specific type of diet could positively influence mental well-being in older adults.

In another study, researchers looked at the relationship between different diets and cognitive decline in Chinese older adults (Chen et al., 2017). The three diets examined were vegetablebased, meat-based, and traditional Chinese diets. The vegetable-based diet was mainly dark-green vegetables and light-colored vegetables cooked with oil. The meat-based diet consisted primarily of red meat, poultry, and roasted meats. The traditional diet was composed mainly of pickled vegetables. The researchers found that the vegetable-based diet helped prevent logical memory-recall decline, the meat-based diet was positively correlated with verbal fluency even in old age, and the traditional diet protected against local memory-recall decline. Another study looked at the relationship between different dietary patterns and cognitive decline in Taiwanese older adults (Tsai, 2014). The participants were categorized into three diets: Western (based primarily on meat and sugar), traditional (based mainly on fruits/vegetables), and healthy (balanced amongst the different food categories, but consisting mainly of fruits/vegetables and fish). The results showed that the participants who followed a healthy dietary pattern displayed less cognitive decline than those who adhered to the Western or traditional diet. The findings of this study were consistent with other studies that explored dietary trends in different countries in that they showed healthy, balanced diets led to better cognitive function or less cognitive decline than 
unbalanced diets (Chen et al., 2017; Luciano et al., 2017).

In contrast, some studies have shown that certain diet types, such as a glycemic-based diet, did not influence older adults' cognitive functioning (Seetharaman et al., 2014). This study's finding was significant because it showed that not all diet types would lead to differences in cognitive function in older adults. It also emphasized the importance of thorough examination of different diet patterns globally and selectively choosing which diet would significantly influence older adults' cognition. Due to worldwide increases in sucrose and fat consumption and the evidence that diet can influence cognitive decline in older adults, studies that examine the relationship between high-sucrose, high-fat diets, and cognition should be prioritized.

The Effect of High-Sucrose Diets on Cognition With an overall increase in dietary sugar consumption, it is imperative to explore the relationship between high-sucrose diets and cognitive abilities. In one study, researchers examined the association between habitual sugar intake and cognitive function in middle-aged and older Puerto Ricans without diabetes (Ye et al., 2011). The study found that participants with higher dietary intake of sugar, sucrose, glucose, and fructose had lower standardized test scores than those with lower intake of the different forms of sugar. This study showed that higher sugar consumption could lead to poorer cognition, measured using seven different neuropsychological assessments. Another study explored the relationship between a high-fructose diet and memory deficits in adult rats (Wu et al., 2014). The findings showed that rats given a $10 \%$ fructose solution instead of water had greater insulin resistance levels in the brain. Insulin plays a vital role in neural plasticity and memory formation; therefore, it was concluded that insulin resistance due to high-fructose consumption led to cognitive impairment.
In another study, the researchers looked at the relationship between sugar and fat consumption in rats (Beilharz et al., 2014). It was shown that rats in both the high-sucrose and high-fat-andhigh-sucrose groups performed poorly compared to the control group. Furthermore, rats given a $10 \%$ sucrose solution in place of water showed greater oxidative stress and more severe hippocampal inflammation than those on a standard diet, high-sucrose diet, or high-fat-andhigh-sucrose group with water. The researchers also found that rats fed sugar water and a standard diet showed greater inflammation and oxidative stress in the brain than those fed a combination of sugar water and a high-fat diet. This study's results are significant because they support the idea that high sucrose intake can damage the brain and impair memory.

Finally, these studies suggest there is a relationship between high-sucrose diets and cognitive function.

\section{The Effects of High-Fat and -Sucrose Diets on Cognition}

With the Western diet on the rise, many researchers have explored the influence of a highfat and -sucrose diet (HFS) on cognitive function in animal models. Kothari et al. (2017) found that the HFS diet could lead to insulin insensitivity and impaired insulin signaling. Because insulin signaling is essential in neurosynaptic plasticity, the researchers suggested that the reduced function of insulin led to cognitive decline in mice. In a different study, Kanoski and Davidson (2010) examined the relationship between highsaturated fat and glucose intake and learning and memory skills in rats, assessed using an eightarmed maze. The study results showed that rats fed with a high saturated fat diet performed significantly worse than those on the standard diet. In other words, there was an obvious memory impairment in the rats that were on a high-saturated fat diet in comparison to those on a balanced diet. These studies support that highsaturated fat diets combined with high sucrose 
content might negatively influence cognitive function in both humans and rats.

\begin{abstract}
Animal Models
According to Iannaccone and Jacob (2009), rat models are often used because of their similarities to the human population. The researchers stated that rats are more appealing than other animal models because they have physiological mechanisms similar to humans. Furthermore, rat models might be the most advantageous to use in cognitive assessments because the physiological systems involved in their learning abilities and memory have been well-studied and documented. Lastly, rat models are more preferred over other animal models because rats can be identically bred within a short time. All these reasons are sufficient to assume that rat models can determine the association between potentially harmful diets and cognitive abilities in older adults. Male rats were used to prevent potential influencing factors linked to female rats' estrous cycles, as well as because previous neurosciencebased studies have found no substantial differences between the sexes. (Beery, 2018; Becker et al., 2016).
\end{abstract}

\section{Hypotheses}

This study predicted that older rats fed a nutritionally balanced standard diet would perform better on a learning abilities task than those fed either a high-sucrose or high-fat diet. Furthermore, we hypothesized that rats fed a high-sucrose diet would perform worse than those fed a high-fat diet. Several studies have shown that a high-sucrose diet alone could lead to oxidative stress and insulin resistance and negatively impact cognitive function (Beilharz et al., 2014; Wu et al., 2014). However, most studies that explored the effects of a high-fat diet on cognitive abilities also used diets with a highsucrose content (Kothari et al., 2017; Kanoski and Davidson, 2010). There was little evidence indicating that only high-fat diet content would negatively impact cognitive function.

\section{Subjects}

Fifteen-month-old male Sprague-Dawley rats (N $=44$; equivalent to about 52 years old in human years) were randomly divided into three groups: balanced $\operatorname{diet}(\mathrm{N}=8)$, high-sucrose $\operatorname{diet}(\mathrm{N}=19)$, and high-saturated fat diet $(\mathrm{N}=17)$ (See Table 1 for a further breakdown of diets). The rats were housed in pairs in a temperature-controlled facility on a standard 12-hr light cycle (lights on at $7 \mathrm{am}$ ). The rats were cared for according to the regulations and guidelines in the Guide for the Care and Use of Laboratory Animals by the National Institute of Health. The Institutional Animal Care and Use Committee (IACUC) at California State University, Fullerton, approved the protocol for this study (Protocol Number: 17R-09) prior to the start of the study.

Table 1.

Nutritional Content of Different Diets

\begin{tabular}{lccc}
\hline & $\begin{array}{c}\text { Protein } \\
(\% \mathrm{kcal})\end{array}$ & $\begin{array}{c}\text { Fat } \\
(\% \mathrm{kcal})\end{array}$ & $\begin{array}{c}\text { Carbohydrates } \\
\text { (sucrose) } \\
(\% \mathrm{kcal})\end{array}$ \\
Standard/Balanced & 29 & 13 & 58 \\
High-Sucrose & 20 & 10 & 70 \\
High-Fat & 16.0 & 37.0 & 36.8 \\
\hline
\end{tabular}

\section{Procedures}

For the first 21 days, the aged rats had access to the respective diets and water ad-libitum. The 21- day threshold was determined by past studies, which suggest that dietary effects occur within 21 days in humans (Maltz, 1960). Because of physiological similarities between rats and 
humans, it was determined that 21 human days, equivalent to around 180 rat days, were sufficient for dietary effects in rats (Sengupta, 2013).

Beginning with the testing day, rats were given water ad-libitum and $10 \mathrm{~g}$ of their respective diets to motivate learning. Their weights were monitored every day to ensure animal safety. The rats were tested consistently at 8 am and fed between one to four hours afterward to prevent them from adjusting to the regular timing of feeding to motivate learning during the testing period.

This study employed a T-maze instrument (Fig. 1), a widely used technique to assess basic learning ability and decision-making in laboratory rodents, which can be generalized to spatial learning and memory in humans (Vorhees \& Williams, 2014).

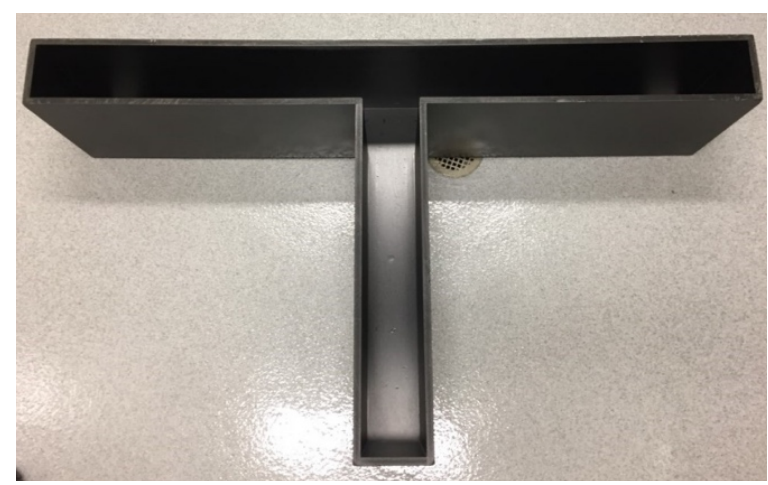

Figure 1. T-maze instrument (arm length: $30 \mathrm{~cm}$, width: $10 \mathrm{~cm}$; height: $20 \mathrm{~cm}$ )

The following procedures were used to train and test the rats in this study; they were based on the protocol used in previous studies assessing decision-making in rodents (Bardgett et al., 2009; Salamone et al., 1994).

Habituation. On the first day of testing, the rats were habituated to the T-maze in groups of two (phase 1). The arms of the maze were not baited, allowing the rats to spend ten minutes in the maze exploring.
Training. Once habituated, reward-baited arms were randomly assigned to each rat and kept constant throughout the training and forward learning phase. The reward was one cheese flavored eCOTRITION Yogies treat made for rats (crude protein: $1.5 \%$, crude fat: $21 \%$, crude fiber: $.5 \%)$. In the training phase (phase 2 ), the un-baited arm was blocked using an arm blocking apparatus, which exposed the rats only to the baited arm. The rats were removed when they consumed the reward or after 2.5 minutes in the maze. After five trials, the baited arm was blocked, and the rats were exposed to the unbaited arm. The rats were removed once they reached the end of the un-baited arm or after 2.5 minutes in the maze ( 5 trials per arm for one day).

Forward Learning. In the forward learning phase (phase 3), the rats were given access to both arms but were removed from the maze after either consuming the reward in the baited arm or reaching the end of the non-baited arm, or after $2.5 \mathrm{~min}$. in the maze ( 10 trials per day for as many days as necessary to reach criterion). The criterion was met once rats correctly chose the reward arm on $90 \%$ of the trials.

Reverse Learning. Once a rat reached the forward learning task criterion, the location of the reward was switched to the opposite arm, and reverse learning began (phase 4). When the rats reached the criterion on this task ( $90 \%$ correct), the study ended.

Rats were tested for an additional seven days if they took considerably longer than their counterparts in any of the learning phases. If the $90 \%$ threshold was not reached, their data were removed from the analysis because they were statistical outliers (data beyond two standard deviations from the norm). These rats were deemed unable to learn the given tasks per study standards.

\section{Measures}

The measures taken in this study were 1) the number of test days it took the rat to reach 
criterion in phase 3, and 2) the number of test days it took the rat to reach criterion in phase 4 . The collected data were analyzed using a twoway repeated measures analysis of variance (ANOVA) to determine the relationship between different diets and their influence on older rats' learning abilities. The dependent variable was the number of days it took for rats to reach the criterion, and the two independent variables were the type of learning (2 levels; forward and reverse), and diet (3 levels; nutritionally balanced standard chow, high-sucrose diet, and highsaturated fat diet).

\section{Results}

Throughout the study, the initial sample size $(\mathrm{N}=$ 44) was reduced to 36 due to attrition. Four rats were dropped from the study (two rats on a standard diet and another two on a high saturatedfat diet): three were euthanized due to tumor development and rapid decrease in weight, and one expired during the testing period. Additionally, the data for one rat on the high sucrose diet and three rats on the high saturatedfat diet were completely removed from the analysis because they were deemed as outliers per our guidelines; they took significantly longer ( $>$ additional seven days) to complete the given tasks compared to the rats in their respective diet groups. As a result, the performance of 36 male 15-month-old Sprague-Dawley rats were analyzed (standard diet: 8; high-sucrose diet: 17; high-saturated fat diet: 11). Due to the small sample size, we applied a 10:1 ratio to each group's individual variances instead of performing a Levene's test after noting the group sizes, and the homogeneity of variance assumption was met (Wang et al., 2016). Mauchly's Test of Sphericity was used to evaluate compound symmetry; the Greenhouse-
Geisser Epsilon value (1.00) met the criteria to assume compound symmetry. In efforts to combat inflated family-wise error, the Bonferroni method was used to adjust alpha. Specifically, a Bonferroni-adjusted alpha level was calculated at $p=.016$, based on an initial Type 1 error rate of .05 used as the starting point.

Before looking at the learning data, ANOVA was used to examine the effects of different diets on rat weights; weights at the start of diet and beginning of testing were used. The results showed no significant effect of diet on rat weights, $F(2)=2.04, p=.15$, suggesting that rats' weight changes were noncontributory to differences in learning.

A (2) x (3) mixed-factor ANOVA was calculated using learning type and diet as the independent variables and days to complete learning as the dependent variable. The findings revealed no significant effect for learning type, $\mathrm{F}(1,33)=$ $1.39, \mathrm{p}=.25$, indicating there was no difference between forward learning $(\mathrm{M}=5.23, \mathrm{SE}=0.85)$ and reverse learning $(\mathrm{M}=4.33, \mathrm{SE}=0.55)$. The subsequent analysis took this into account, and the impact of different diets on overall learning abilities was analyzed by collapsing the data across both levels of learning. There was a significant effect of diet on days to complete learning, $\mathrm{F}(2,33)=14.74, \mathrm{p}<.05$ (See Fig. 2). Post-hoc tests indicated that rats fed either highsugar diet $(\mathrm{M}=5.53, \mathrm{SE}=0.65)$ or high-fat diet $(\mathrm{M}=6.18, \mathrm{SE}=0.87)$ took significantly more time to reach criterion than rats fed control diet $(\mathrm{M}=2.63, \mathrm{SE}=0.58)$; however, the high-sugar and high-fat groups were not different from each other (see Table 2). There was no significant interaction between learning type and diet on days to complete learning, $\mathrm{F}(2,33)=1.50, \mathrm{p}=$ .24 . 
Table 2.

Numerical Values Related to Completing Task

\begin{tabular}{|c|c|c|c|c|}
\hline & & & Mean Number of Days & \\
\hline & & Range of Number of Days & & Standard Error \\
\hline & Standard Diet & $1-5$ & 2.13 & 0.55 \\
\hline$\stackrel{900}{J}$ & High-Sucrose Diet & $2-13$ & 6.12 & 0.79 \\
\hline 尝 & $\begin{array}{l}\text { High-Saturated Fat } \\
\text { Diet }\end{array}$ & $2-15$ & 7.25 & 1.12 \\
\hline & Standard Diet & $1-6$ & 3.13 & 0.61 \\
\hline $\mathscr{\infty}$ & High-Sucrose Diet & $2-9$ & 4.94 & 0.50 \\
\hline 氞 & $\begin{array}{l}\text { High-Saturated Fat } \\
\text { Diet }\end{array}$ & $2-11$ & 5.42 & 0.70 \\
\hline
\end{tabular}

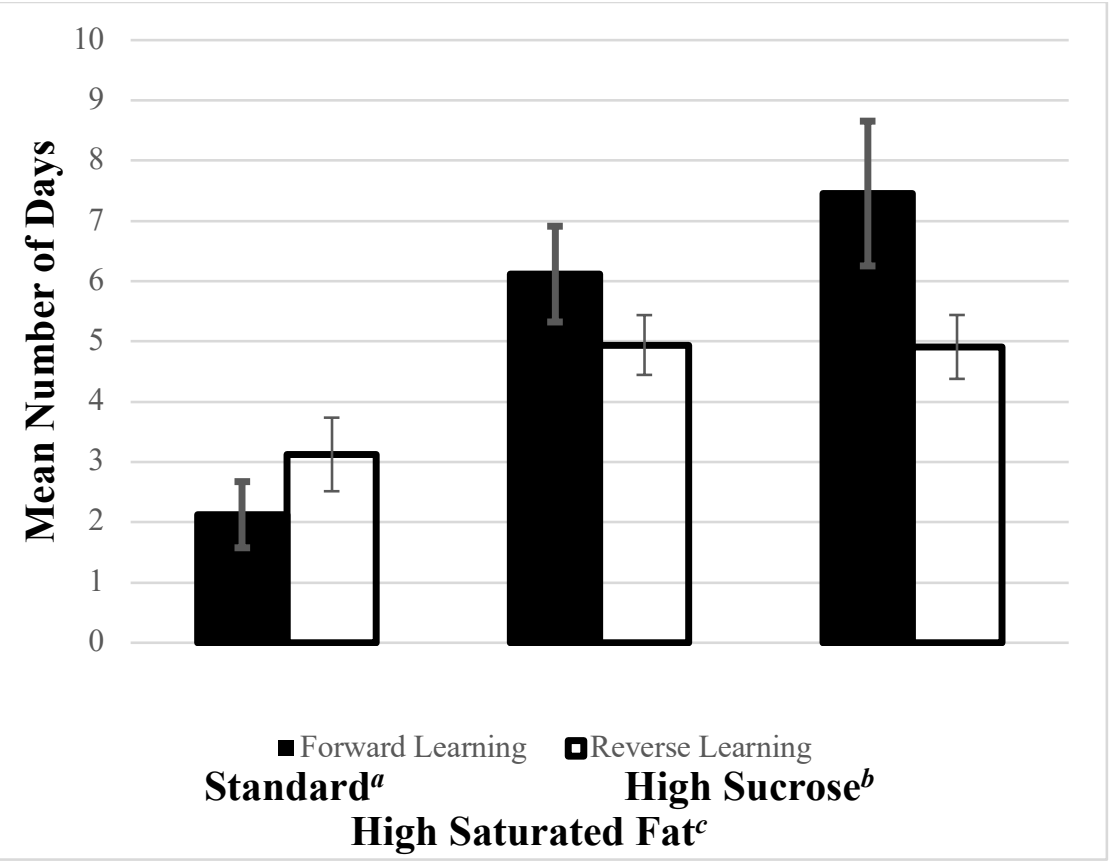

Figure 2. Differential Effects of Different Diets on Learning Abilities in Old Male Rats

Note. Animals were on three different prior to testing: standard ( $a: \mathrm{N}=8)$, high sucrose $(b: \mathrm{N}=17)$, high saturated fat $(c: \mathrm{N}=11)$, and learning abilities were tested using a T-maze. No significant effect of diet was found between forward learning and reverse learning, $\mathrm{F}(2,33)=1.50, \mathrm{p}=.24$, so subsequent analysis combined forward learning and reverse learning. There was significant impact of diets on overall learning F $(2,33)=14.74, \mathrm{p}<.05$. Post-hoc tests showed that animals on high sucrose $\operatorname{diet}(\mathrm{M}=5.53, \mathrm{SE}=0.65)$ or high-fat diet $(\mathrm{M}=6.18, \mathrm{SE}=0.87)$ performed significantly worse than those on standard diet; difference between the two specialized diets were not significant. 


\section{Discussion}

There was no significant effect found between forward learning and reverse learning across the different diet groups. It is possible that the diets studied had a general impact on learning as opposed to specific types of learning, which is consistent with past findings (Luciano et al., 2017; Samieri et al., 2015). In one study, it was found that Western diet (high-fat and highsucrose diet) led to an increase in brain blood barrier transport and permeability, in addition to brain inflammation regardless of the rodents' genotype compared to before the diet change (Rutkowsky et al., 2018). The researchers concluded that this could lead to a general increase in oxidative stress in the brain resulting in impaired cognition.

As predicted, rats on a standard diet performed better in cognitive learning tasks than those on either a high-sucrose or high-saturated fat diet. The study results are consistent with previous findings that diets high in sucrose or fat impair cognitive function ( $\mathrm{Ye}$ et al., 2011; Wu et al., 2014; Beilharz et al., 2014). This may be due to changes in the brain. Previous findings showed that a combination of high-saturated fat and highsucrose diets led to poorer hippocampal-related memory function in humans using the verbal paired-associate test, which is known to be a hippocampal-dependent process to assess hippocampal-related memory (Attuquayefio et al., 2016). This implies some hippocampal changes associated with the high-sucrose and high-saturated fat diet intake in humans. Greenwood and Winocur (1996) found that young rats on a high-saturated fat diet showed greater learning impairment than those on a standard diet. The researchers concluded that the impairment was directly related to the amount of saturated fatty acid intake; however, the exact mechanism by which dietary intake of saturated fatty acids influences neural rewiring is unknown. Moreover, studies suggest possible neural rewiring in different parts of the brain, whether region-specific or mechanism-specific
(Attuquayefio et al., 2016; Greendwood \& Winocur, 1996). Thus, it implies that rats on a high-sucrose and a high-saturated fat diet might have developed neural connections in different patterns or at a slower rate than those on a nutritionally balanced diet.

Findings from other studies indicate that the difference in cognitive abilities amongst the three different diets might be due to oxidative stress and metabolic syndrome. Beilharz et al. (2016) found that a high-sucrose diet and a combination of high-sucrose and high-saturated fat diet triggered an increase in oxidative stress and induced brain inflammation, which correlated to place recognition impairment in rats. The researchers further examined the effects of a highsucrose, high-saturated fat diet on selective areas of the brain. They found that the hippocampal region showed higher inflammation compared to other regions of the brain. Moreover, Agrawal and Gomez-Pinilla (2012) found that rats on high-sucrose diets worked through a Barnes-type maze at a slower rate than those on a standard diet. The effects of a high-sucrose diet were further tested when omega-3 fatty acids, otherwise known as "healthy" fat, were added to the diet; they saw that omega- 3 fatty acid addition partially reversed the exacerbated cognitive abilities that were induced by a high-sucrose diet. The researchers concluded that high-sucrose intake composed mainly of fructose was positively correlated to the amount of insulin resistance, which could lead to an increase in triglyceride levels in the body, including the brain. Triglycerides have been found to negatively affect memory function if present in the brain ventricles (Farr et al., 2008). The researchers indicate that oxidative stress and brain inflammation could lead to the discrepancies in learning abilities between rats on a nutritionally healthy balanced diet and rats on specific diets such as a high-fat and high-sucrose diet.

It was surprising to find that the learning abilities between rats on a high-sucrose and high-saturated 
fat diets are not significantly different because previous studies have found that a high-sucrose diet has been associated with memory impairment more so than a high-saturated fat diet (Ye et al., 2011; Wu et al., 2014). Interestingly, data analysis of present study showed the exact opposite. Learning took longer for rats on a highsaturated fat diet than those on a high-sucrose diet (See Fig. 2). As previously mentioned, only a few studies have looked at the sole effect of a highsaturated fat diet on learning abilities, but in conjunction with a high-sucrose diet (Beilharz et al., 2014; Beilharz et al., 2016). As such, it might be worthwhile to explore further the possible differences between the two diets on learning abilities.

In conclusion, the current study demonstrated that both a high-sucrose and a high-saturated fat diet negatively influenced learning abilities in geriatric rats compared to a nutritionally balanced diet. The findings suggest that high-sucrose or high-saturated fat diets are similarly detrimental to cognitive health in later years. The findings of this study could be used to develop suitable dietary plans for older adults in residential environments, nursing care facilities, assisted living homes, and senior centers to ensure that their dietary preferences include foods that are low in both sugars and saturated fat. Implementation of such diets may assist older adults in aging successfully.

\section{Limitations and Future Studies}

This study has a few limitations. Attrition is an unfortunate occurrence with live rat studies but is particularly common in older rats. The resulting smaller sample size due to age-related attrition and data exclusion might have reduced the generalizability of the study. Furthermore, the duration of diet might have also influenced the results of the study. For future studies, we recommend a large sample size and longer diet duration.

\section{Conclusion}

The study's findings revealed that different diets have varying effects on older rats' cognitive abilities and can be used to raise awareness of the enormous possible effects of diet on cognitive health in older adults. These findings may be used to develop an optimal dietary plan for older adults residing in assisted living, skilled nursing facilities, and the Meals On Wheels recipients.

\section{References}

Agrawal, R., \& Gomez-Pinilla, F. (2012). 'Metabolic syndrome' in the brain: deficiency in omega-3 fatty acid exacerbates dysfunctions in insulin receptor signaling and cognition. The Journal of Physiology,590(10), 2485-99. doi: 10.1113/jphysiol.2012.230078

Attuquayefio, T., Stevenson, R., Boakes, R., Oaten, M., Yeomans, M., Mahmut, M., \& Francis, H. (2016). A high-fat high-sugar diet predicts poorer hippocampal-related memory and a reduced ability to suppress wanting under satiety. Journal of Experimental Psychology: Animal Learning and Cognition, 42(4), 415-428. doi: 10.1037/xan0000118

Bardgett, M., Depenbrock, M., Downs, N., Points, M., \& Green, L. (2009). Dopamine modulates effortbased decision making in rats. Behavioral Neuroscience, 123(2), 242-251. doi: 10.1037/a0014625

Becker, J., Prendergast, B., \& Liang, J. (2016). Female rats are not more variable than male rats: a metaanalysis of neuroscience studies. Biology of Sex Differences, 7(1). doi: 10.1186/s13293-016$\underline{0087-5}$

Beery, A. (2018). Inclusion of females does not increase variability in rodent research studies. Current Opinion in Behavioral Sciences, 23, 143-149. doi: 10.1016/j.cobeha.2018.06.016 
Beilharz, J., Maniam, J., \& Morris, M. (2014). Short exposure to a diet rich in both fat and sugar or sugar alone impairs place, but not object recognition memory in rats. Brain, Behavior, and Immunity, 37, 134-141. doi: 10.1016/j.bbi.2013.11.016

Beilharz, J., Maniam, J., \& Morris, M. (2016). Short-term exposure to a diet high in fatand sugar, or liquid sugar, selectively impairs hippocampal-dependent memory, with differential impacts on inflammation. Behavioural Brain Research, 306, 1-7. doi: 10.1016/j.bbr.2016.03.018

Bell, R., Davis, C., Tooze, J., Vitolins, M., Smith, S., \& Arcury, T. et al. (2007).Consumption of sugarand fat-modified foods among rural older adults. Journal of Nutrition for the Elderly, 25(3-4), 2340. doi: $10.1300 / \mathrm{j} 052 \mathrm{v} 25 \mathrm{n} 03 \quad 03$

Bowling, A., \& Dieppe, P. (2005). What is successful ageing and who should define it?. BMJ, 331(7531), 1548-1551. doi: $10.1136 / \mathrm{bmj} .331 .7531 .1548$

Chen, Y., Jung, C., Chen, J., Chiou, J., Chen, T., \& Chen, Y. et al. (2017). Association ofdietary patterns with global and domain-specific cognitive decline in Chinese elderly. Journal of the American Geriatrics Society, 65(6), 1159-1167. doi: 10.1111/jgs.14741

Farr, S., Yamada, K., Butterfield, D., Abdul, H., Xu, L., \& Miller, N. et al. (2008). Obesity and Hypertriglyceridemia Produce Cognitive Impairment. Endocrinology, 149(5), 2628-2636. doi: 10.1210/en.2007-1722

Greenwood, C. E. (1995). Cognitive impairment in rats fed high-fat diets: A specific effect ofsaturated fatty-acid intake. American Psychological Association 110(4), 451-459. doi: 10.1037/07357044.110.3.451.

Iannaccone, P. M., \& Jacob, H. J. (2009). Rats! Disease Models \& Mechanisms, 2(5-6),206-210. doi: 10.1242/dmm.002733

Jacob, M., Yee, L., Diehr, P., Arnold, A., Thielke, S., \& Chaves, P. et al. (2016). Can ahealthy lifestyle compress the disabled period in older adults?. Journal of the American Geriatrics Society, 64(10), 1952-1961. doi: 10.1111/jgs. 14314

Kanoski, S., \& Davidson, T. (2010). Different patterns of memory impairmentsaccompany short- and longer-term maintenance on a high-energy diet. Journal of Experimental Psychology: Animal Behavior Processes, 36(2), 313-319. doi: 10.1037/a0017228

Kothari, V., Luo, Y., Tornabene, T., O'Neill, A., Greene, M., Geetha, T., \& Babu, J. (2017). High fat diet induces brain insulin resistance and cognitive impairment in mice. Biochimica Et Biophysica Acta (BBA) - Molecular Basis of Disease, 1863(2), 499-508. doi: 10.1016/j.bbadis.2016.10.006

Luciano, M., Corley, J., Cox, S., Valdés Hernández, M., Craig, L., \& Dickie, D. et al. (2017). Mediterranean-type diet and brain structural change from 73 to 76 years in a Scottish cohort. Neurology, 88(5), 449-455. doi: 10.1212/wnl.0000000000003559

Maltz, M. (1960). Psycho-cybernetics: A new way to get more living out of life. Simon \& Schuster.Poor Nutrition. Cdc.gov. (2021). Retrieved 5 March 2021, from https:/www.cdc.gov/chronicdisease/resources/publications/factsheets/nutrition.htm.

Rutkowsky, J., Lee, L., Puchowicz, M., Golub, M., Befroy, D., \& Wilson, D. et al. (2018). Reduced cognitive function, increased blood-brain-barrier transport and inflammatory responses, and altered brain metabolites in LDLr -/-and C57BL/6 mice fed a western diet. PLOS ONE, 13(2), e0191909. doi: 10.1371/journal.pone.0191909

Salamone, J., Cousins, M., \& Bucher, S. (1994). Anhedonia or anergia? Effects ofHaloperidol and nucleus accumbens dopamine depletion on instrumental response selection in a T-maze cost/benefit procedure. Behavioural Brain Research, 65(2), 221-229. doi:10.1016/01664328(94)90108-2 
Samieri, C., Pelletier, A., Barul, C., Féart, C., Helmer, C., \& Bernard, C. et al. (2015).The Mediterranean diet and preservation of brain connectivity in older subjects. Alzheimer's \& Dementia, 11(7), 454P455. doi: 10.1016/j.jalz.2015.06.463

Seetharaman, S., Andel, R., McEvoy, C., Dahl Aslan, A., Finkel, D., \& Pedersen, N. (2014). Blood glucose, diet-based glycemic load and cognitive aging among dementia-free older adults. The Journals of Gerontology Series A: Biological Sciences and Medical Sciences, 70(4), 471-479. doi: $10.1093 /$ gerona/glu135

Sengupta P. (2013). The Laboratory Rat: Relating Its Age With Human's. International Journal of Preventive Medicine, 4(6), 624-630. https://pubmed.ncbi.nlm.nih.gov/23930179/

Stephan, B., Wells, J., Brayne, C., Albanese, E., \& Siervo, M. (2010). Increased fructoseintake as a risk factor for dementia. The Journals of Gerontology Series A: Biological Sciences and Medical Sciences, 65A(8), 809-814. doi: 10.1093/gerona/glq079

Tsai, H. (2014). Dietary patterns and cognitive decline in Taiwanese aged 65 years and older. International Journal of Geriatric Psychiatry, 30(5), 523-530. doi: 10.1002/gps.4176

Vorhees, C., \& Williams, M. (2014). Assessing Spatial Learning and Memory in Rodents. ILAR Journal, 55(2), 310-332. doi: 10.1093/ilar/ilu013

Wang, Y., Rodríguez de Gil, P., Chen, Y., Kromrey, J., Kim, E., \& Pham, T. et al. (2016). Comparing the Performance of Approaches for Testing the Homogeneity of Variance Assumption in One-Factor ANOVA Models. Educational and Psychological Measurement, 77(2), 305-329. doi: $10.1177 / 0013164416645162$

Wu, H., Ren, L., Zhou, X., \& Han, D. (2014). A high-fructose diet induces hippocampalinsulin resistance and exacerbates memory deficits in male Sprague-Dawley rats. Nutritional Neuroscience, 18(7), 323-328. doi: 10.1179/1476830514y.0000000133

Ye, X., Gao, X., Scott, T., \& Tucker, K. (2011). Habitual sugar intake and cognitive function among middle-aged and older Puerto Ricans without diabetes. British Journal of Nutrition, 106(09), 1423-1432. doi: $10.1017 / \mathrm{s} 0007114511001760$

Corresponding Author Information

Ji Min Lee

Address: 7400 Artesia Blvd.

Apt. 1706

Buena Park, CA 90621

Phone Number: 714-292-8789

Email: jiminius@gmail.com 